\author{
SANDIA REPORT \\ SAND2006-6955 \\ Unlimited Release \\ Printed November 2006
}

\title{
Viral Vectors for Gene Modification of Plants as Chem/Bio Sensors
}

Monica Manginell, Dulce Arango, Patricia Dolan, Jason Harper, Susan Brozik

Prepared by

Sandia National Laboratories

Albuquerque, New Mexico 87185 and Livermore, California 94550

Sandia is a multiprogram laboratory operated by Sandia Corporation,

a Lockheed Martin Company, for the United States Department of Energy's

National Nuclear Security Administration under Contract DE-AC04-94AL85000.

Approved for public release; further dissemination unlimited. 
Issued by Sandia National Laboratories, operated for the United States Department of Energy by Sandia Corporation.

NOTICE: This report was prepared as an account of work sponsored by an agency of the United States Government. Neither the United States Government, nor any agency thereof, nor any of their employees, nor any of their contractors, subcontractors, or their employees, make any warranty, express or implied, or assume any legal liability or responsibility for the accuracy, completeness, or usefulness of any information, apparatus, product, or process disclosed, or represent that its use would not infringe privately owned rights. Reference herein to any specific commercial product, process, or service by trade name, trademark, manufacturer, or otherwise, does not necessarily constitute or imply its endorsement, recommendation, or favoring by the United States Government, any agency thereof, or any of their contractors or subcontractors. The views and opinions expressed herein do not necessarily state or reflect those of the United States Government, any agency thereof, or any of their contractors.

Printed in the United States of America. This report has been reproduced directly from the best available copy.

Available to DOE and DOE contractors from

U.S. Department of Energy

Office of Scientific and Technical Information

P.O. Box 62

Oak Ridge, TN 37831

Telephone: $\quad$ (865) 576-8401

Facsimile: $\quad$ (865) 576-5728

E-Mail: $\quad$ reports@adonis.osti.gov

Online ordering: http://www.osti.gov/bridge

Available to the public from

U.S. Department of Commerce

National Technical Information Service

5285 Port Royal Rd.

Springfield, VA 22161

Telephone: $\quad$ (800) 553-6847

Facsimile: (703) 605-6900

E-Mail: $\quad$ orders@ntis.fedworld.gov

Online order: $\quad$ http://www.ntis.gov/help/ordermethods.asp?loc=7-4-0\#online

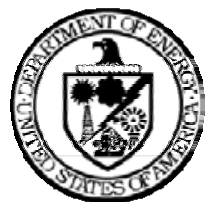


SAND2006-6955

Unlimited Release

Printed November 2006

\title{
Viral Vectors for Gene Modification of Plants as Chem/Bio Sensors
}

\author{
Monica Manginell, Dulce Arango, Patricia Dolan, Jason Harper, Susan Brozik \\ Biosensors and Nanomaterials \\ Sandia National Laboratories \\ P.O. Box 5800 \\ Albuquerque, NM 87185
}

\begin{abstract}
Chemical or biological sensors that are specific, sensitive, and robust allowing intelligence gathering for verification of nuclear non-proliferation treaty compliance and detouring production of weapons of mass destruction are sorely needed. Although much progress has been made in the area of biosensors, improvements in sensor lifetime, robustness, and device packaging are required before these devices become widely used. Current chemical and biological detection and identification techniques require less-than-covert sample collection followed by transport to a laboratory for analysis. In addition to being expensive and time consuming, results can often be inconclusive due to compromised sample integrity during collection and transport. We report here a demonstration of a plant based sensor technology which utilizes mature and seedling plants as chemical sensors. One can envision genetically modifying native plants at a site of interest that can report the presence of specific toxins or chemicals.
\end{abstract}

In this one year project we used a developed inducible expression system to show the feasibility of plant sensors. The vector was designed as a safe, non-infectious vector which could be used to invade, replicate, and introduce foreign genes into mature host plants that then allow the plant to sense chem/bio agents. The genes introduced through the vector included a reporter gene that encodes for green fluorescent protein (GFP) and a gene that encodes for a mammalian receptor 
that recognizes a chemical agent. Specifically, GFP was induced by the presence of 17- $\beta$ Estradiol (estrogen). Detection of fluorescence indicated the presence of the target chemical agent.

Since the sensor is a plant, costly device packaging development or manufacturing of the sensor were not required. Additionally, the biological recognition and reporting elements are maintained in a living, natural environment and therefore do not suffer from lifetime disadvantages typical of most biosensing platforms. Detection of the chem/bio agent reporter (GFP) can be detected only at a specific wavelength. 


\section{Contents}

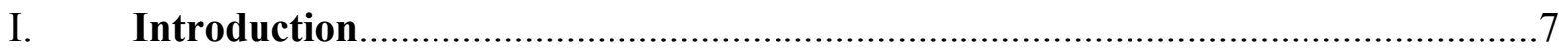

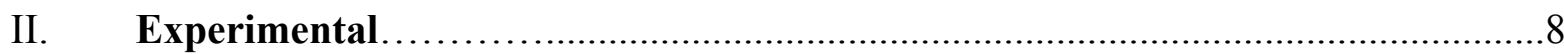

A. Transformation of Agrobacterium strain EHA105 with the pER8-GFP

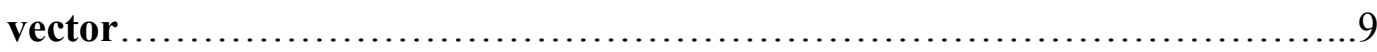

B. Plant growth and seed harvesting .............................................................10

C. Transformation of Arabidopsis using Agrobacterium mediated transformation ...........................................................

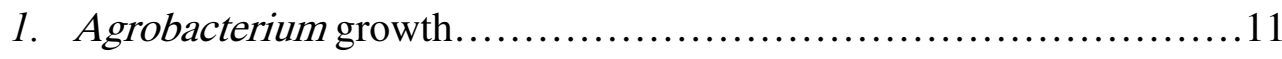

2. Transformation of Arabidopsis........................................11

3. Harvesting and storing seeds....................................11

D. Selection and GFP induction of transformed plants......................12

1. Sterilization of seeds........................................... 12

2. Growth on solid media using antibiotic selection......................12

3. Induction and expression of green fluorescent protein.................12

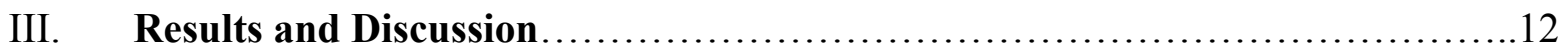

A. Transformation of Agrobacterium strain EHA105 with pER8-GFP...........12

B. Selection of hygromycin resistant seedlings...............................13

C. GFP reporter assay ....................................................... 14

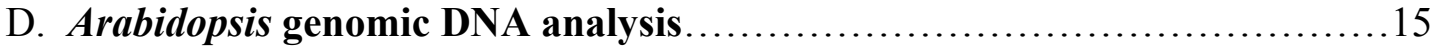

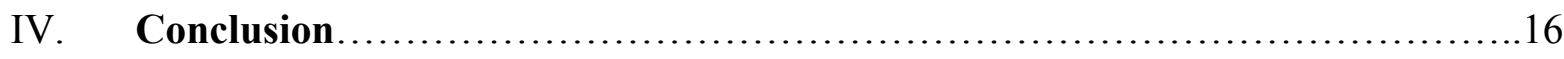

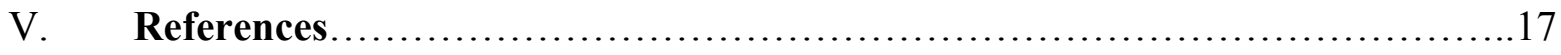




\section{Figures}

Figure 1 GFP produced by ZYMV infected cucumber plant under UV light..............

Figure 2 GFP expression in genetically engineered pine cells in response to induction in the glucocorticoid, dexamethasone..........................................

Figure 3 Verification of pER8-GFP plasmid in Agrobacterium strain EHA105............13

Figure 4 Hygromycin selection of a pER8-GFP transgenic plant .......................14

Figure 5 Top: Transformed Arabidopsis induced with estradiol

Bottom: Transformed Arabidopsis-not induced (modified medium + hygromycin), Non-Transformed Arabidopsis (modified medium), NonTransformed Arabidopsis (modified medium + hygromycin +estradiol).........14

Figure 6 Amplified GFP from Arabidopsis genomic DNA..............................15 


\section{Introduction}

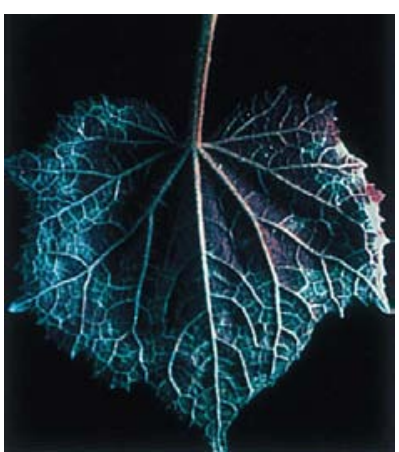

Figure 1. GFP produced by ZYMV infected cucumber plant under UV light (1).
The greatest challenges to developing genetically modified plant sensors for chemical detection are 1) the production of viable expression systems that only respond to specific inducers followed by activation of the target genes and 2) successful inoculation of seedling and mature plants. Several chemical inducible systems have been developed. Arazi et al. ${ }^{1}$ designed a plant vector that is neither harmful to the host plants nor can it be transferred to other plants. The vector is a genetically modified zucchini yellow mosaic potyvirus (ZYMV) that infects plants in the cucurbit species such as

cucumber, squash, and watermelon. A single amino acid modification to the virus prevents transfer of genes to other plants. Insertion of the gene for green fluorescent protein (GFP) into the viral vector showed significant expression in infected squash and cucumber leaves, stems, roots, and edible fruit (Figure 1). Additionally, plants inoculated with the ZYMV vector showed mild or no symptoms of viral infection and grew at similar rates to non-infected control plants. Modification of the ZYMV vector such that GFP is expressed only in the presence of a specific chemical or biological agent has not been demonstrated.

Successful introduction of chemically-inducible gene expression promoters from prokaryotes, insects, and mammals into plant vectors has also been reported. Chemicals that have been reported to regulate foreign gene expression include the antibiotic tetracycline, ethanol, copper, the insecticide methoxyfenozide, and the steroids estrogen, triamcinolone, and dexamethasone. A GVG-regulated expression vector has shown particular promise in plant systems ${ }^{2}$. Gene promotion via the GVG
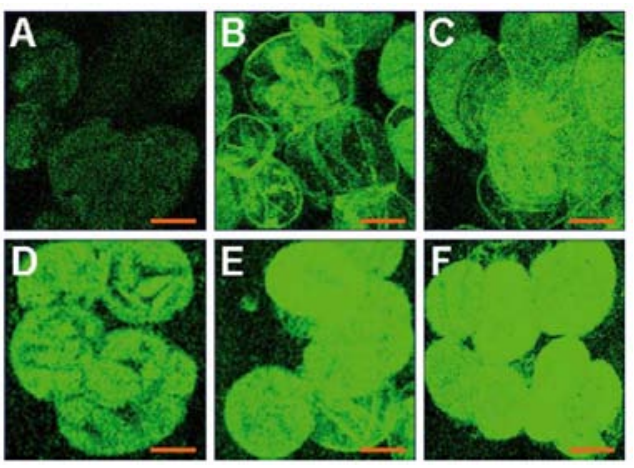

Figure 2. GFP expression in genetically engineered pine cells in response to induction in the glucocorticoid, dexamethasone $(5 \mathrm{mg} / \mathrm{l})$ for $0 \mathrm{~h}(\mathrm{~A}), 12 \mathrm{~h}$ (B), $24 \mathrm{~h}(\mathrm{C}), 48 \mathrm{~h}(\mathrm{D}), 72 \mathrm{~h}(\mathrm{E})$, and $96 \mathrm{~h}$ (F); (2). system is based on the response of a mammalian (rat) receptor to glucocorticoids, a family of steroids that are involved in glucose metabolism. Advantages of this system include 1) glucocorticoid hormones have no naturally occurring analogues in plant cells, 2) induction successfully occurs at micromolar levels and has no 
negative affect on engineered Virginia pine cells (Figure 2), and 3) control of GFP expression was very tight.

Zuo et al. developed an estrogen receptor based inducible system for use in transgenic tobacco and Arabidopsis plants ${ }^{3}$. Specifically, they inserted a cDNA encoding the GFP into the expression cassette of the pER8 vector. This vector also contained the regulatory region of the human estrogen receptor. The pER8-GFP vector was transformed into plants and upon induction by estradiol, GFP was expressed. This expression system has the same advantages as that of the GVG system; i.e., it is tightly regulated and highly inducible in transgenic plants. The activator is strictly regulated by estradiol, capable of stimulating GFP expression with undetectable transactivating activity in the absence of the chemical inducer. We obtained the plasmid, pER8GFP (developed by Zuo et al. at Rockefeller University) and report here the successful transformation of this plasmid into Arabidopsis thaliana host plants and the expression of the GFP reporter protein in response to 17- $\beta$-Estradiol (estrogen).

\section{Changes to the Project}

We initially chose a genetically modified zucchini yellow mosaic potyvirus (ZYMV), as the noninfectious viral vector to produce GFP in plants such as cucumber and squash. Our goal for this project remained the same; we demonstrated that we could successfully inoculate mature and seedling plants with recombinant DNA as a method of developing mature plants into chem/bio sensors. However rather than using a viral vector and a plant such as squash or cucumber which is considered a cash crop, we obtained the plasmid pER8-GFP. This plasmid is safe and was used to transform Arabidopsis thaliana host plants. Arabidopsis thaliana was used instead of a cash crop as an environmental precaution.

\section{Experimental}

We demonstrated the feasibility of using inoculated plants as chemical sensors by exposing the plants to varying concentrations of estradiol via moisture in the soil and an aerosol. The response of the plants to the target analyte was monitored by measuring green fluorescence of the leaves upon exposure to a UV lamp and laser excitation under a light microscope. 


\section{(A) Transformation of Agrobacterium strain EHA105 with the pER8-GFP vector.}

Transformation was conducted by following the Q-biogene protocol recommended for Agrobacterium transformation:

1. Agrobacterium strain EHA105 was grown on plates as single colonies. Medium was prepared and the appropriate antibiotics (rifampin to select for a chromosomal EHA105 marker and spectinomycin to select for the pER8-GFP vector) were added once the solutions had cooled to room temperature. Rifampin is light sensitive and therefore the medium was stored in the dark.

2. An Agrobacterium colony, obtained from a plate no older than two weeks, was placed in a $50 \mathrm{ml}$ conical tube and was inoculated with $20 \mathrm{ml}$ of Agrobacterium medium. The colony was incubated $18-38$ hours at $28-30^{\circ} \mathrm{C}$ to an $\mathrm{OD}_{600}$ of $1.0-2.0$ in a shaker at 200 rpm.

3. $5 \mathrm{ml}$ aliquots of Agrobacterium culture were placed into $15 \mathrm{ml}$ falcon tubes and centrifuged at $3500 \mathrm{x} \mathrm{g}$ for $5 \mathrm{~min}$ at room temperature or $4^{\circ} \mathrm{C}$ and the supernatant removed. Each $5 \mathrm{ml}$ cell pellet was resuspended in $90 \mu \mathrm{l}$ of ice-cold Agrobacterium Transformation Solution with $500 \mathrm{ng}$ plasmid DNA and mixed gently. The sample was submerged into liquid nitrogen for one minute and then transferred to a $30^{\circ} \mathrm{C}$ water bath for 5 minutes. Finally $0.9 \mathrm{ml}$ of prepared Agrobacterium medium was added to the tube and the samples were incubated in a $28-30^{\circ} \mathrm{C}$ shaker at $200 \mathrm{rpm}$ for 30 minutes to 3 hours.

NOTE: For optimal results, transformed cells were allowed to recover 3 hours.

4. $20 \mu \mathrm{l}$ of the recovered cells were plated onto selective Agrobacterium medium agar plates using Roll \&Grow ${ }^{\circledR}$ Plating Beads. The plates were inverted and incubated at 28$30^{\circ} \mathrm{C}$ for $48-72$ hours.

5. In order to confirm the uptake of pER8-GFP by Agrobacterium, DNA was extracted from the EHA105 transformants and transformed into an E. coli cell line (X-L10-Gold ${ }^{\circledR}$ 
Ultracompetent Cells (Strategene, La Jolla, CA)). These new E. coli transformants allowed for easier manipulation of pER8-GFP for further analysis.

6. The transformation of Agrobacterium was verified by restriction enzyme digest analysis. Plasmid DNA was cut with enzymes XhoI and SpeI (New England BioLabs, USA). Agrobacterium strains with intact plasmid were stored frozen. A negative control was conducted using competent Agrobacterium without added pER8 DNA and pER8 transformed cells were selected on plates with Agrobacterium medium that contained both rifampin and spectinomycin.

\section{(B) Plant growth and seed harvesting.}

Columbia ecotype Arabidopsis thaliana seeds were obtained from LEHLE Seeds (Round Rock, Texas) and were cultivated as follows:

1. Approximately $1 / 2$ " PM-15 potting mixture (LEHLE Seeds) was added to 8 oz. Styrofoam cups with holes in the bottom. Three pellets of 5-19-12 slow release fertilizer (LEHLE Seeds) were added and then the cups were filled to $\sim 1 / 4$ " from the top and lightly compressed. Approximately half a cup of water was added to the soil and allowed to absorb for 30 minutes. The cups were then flood sub-irrigated for another 30 minutes.

2. Approximately 7 seeds were evenly distributed on top of the soil and $1 / 4$, fresh water was added to the tray. The cups were covered securely with plastic wrap and foil and then chilled at $4^{\circ} \mathrm{C}$ for 48 hours in the dark to synchronize growth, improve germination rate and break dormancy (stratification).

3. After 48 hours the foil was removed and plants were grown under $410 \mu \mathrm{moles} / \mathrm{m}^{2} / \mathrm{sec}$ photons at $25^{\circ} \mathrm{C}$ during the day and $21^{\circ} \mathrm{C}$ at night. Photons and temperature were measured with a quantum meter 20 inches from the light source. Once seedlings were established the seal around the plastic was removed and the loosened plastic remained over the seedlings for one additional day of growth. 
4. After approximately 10 days of growth the plants were thinned to 1-2 per cup and Ortho Garden Disease Control was sprayed on the soil surface. The dilution and directions for broccoli on the bottle were used. Plants were watered every $3^{\text {rd }}$ and $4^{\text {th }}$ day by adding $1 / 4$ " water to the tray and the first bolts were clipped off as soon as they appeared to promote the growth of more inflorescences.

\section{(C) Transformation of Arabidopsis using Agrobacterium mediated transformation.}

1. Agrobacterium growth. Agrobacterium containing the pER8-GFP vector was grown to stationary phase $\left(\mathrm{OD}_{600} \sim 2.0\right)$ in $50 \mathrm{ml}$ of $\mathrm{LB}$ media plus spectinomycin $(100 \mu \mathrm{g} / \mathrm{ml})$ and rifampicin $(10 \mu \mathrm{g} / \mathrm{ml})$. The cultures were grown overnight at $28^{\circ} \mathrm{C}$ with shaking at 200 rpm. The overnight culture was added to a total volume of $300 \mathrm{ml}$ of fresh LB medium containing the same antibiotic concentrations and again grown to stationary phase $\left(\mathrm{OD}_{600}\right.$ 2.0). Cells were harvested by centrifugation at $5500 \mathrm{x} \mathrm{g}$ for 20 minutes and then resuspend in $50 \mathrm{ml}$ of an infiltration medium made of $0.5 \mathrm{X}$ MS salts, $5 \%$ sucrose $(\mathrm{w} / \mathrm{v})$, and $0.05 \%$ silwett $\mathrm{L}-77(\mathrm{v} / \mathrm{v})$. This cell suspension produced an $\mathrm{OD}_{600}$ of approximately 1.8 .

2. Transformation of Arabidopsis. The transformation technique was based on the "Floral Dropper" method by Martinez-Trujillo et al. ${ }^{4}$. Plants with inflorescences about $5 \mathrm{~cm}$ tall were selected for first inoculation. Agrobacterium inoculum was applied drop-by-drop to all closed flower buds using a micropipette and cups were subsequently covered with plastic wrap to retain high humidity and placed in the dark at room temperature for $\sim 10$ hours. Closed flower buds were inoculated in this way three more times every 4 days. Plants continued to grow under $410 \mu \mathrm{moles} / \mathrm{m}^{2} / \mathrm{sec}$ photons at $25^{\circ} \mathrm{C}$ during the day and $21{ }^{\circ} \mathrm{C}$ at night with watering every third and fourth day until the first silique turned brown. After the first silique turned brown watering was discontinued.

3. Harvesting and storing seeds. After the entire inflorescence browned, seeds were harvested by cutting the stem just above the rosette. Seeds were placed in an envelope and the silique were crushed against the envelope and filtered through a hand held sieve (\#35 or 40) onto a piece of paper. The seeds were allowed to air dry for $\sim 1$ week and 
then stored in ArabiKeeper (LEHLE Seeds) envelopes and storage boxes at $4^{\circ} \mathrm{C}$. Seeds and storage envelopes were weighed before refrigeration or freezing to monitor moisture content.

\section{(D) Selection and GFP induction of transformed plants.}

1. Sterilization of seeds. Seeds were soaked 30 minutes in water, allowed to settle, and then the water was removed. $95 \% \mathrm{EtOH}$ was added, gently mixed for 5 minutes, and then removed. Seeds were transferred to a solution of $10 \%$ commercial Clorox (i.e. $0.525 \%$ sodium hypochlorite) plus $0.1 \%$ Tween - 20 for exactly 5 minutes. Seeds were rinsed 5 times with sterile water and then maintained in $0.1 \%$ agar. Sterilized seeds were plated within 24 hours.

2. Growth on solid media using antibiotic selection. To select for transformed plants sterilized seeds were plated on modified MS medium plus hygromycin (1 X MS Salts, $1 \%$ Sucrose (w/v), 0.8\% Agar (w/v), 1 X MS Vitamin Solution (Sigma 1000 X), 0.5 g/L MES pH 5.7, $20 \mu \mathrm{g} / \mathrm{ml}$ hygromycin) and sealed with microporous surgical tape. The plates were placed directly into the growth environment as previously described. Plants that grew in the presence of hygromycin were selected as pER8-GFP transformants and were transferred to soil and grown to maturity. The seeds were harvested and stored.

3. Induction and expression of green fluorescent protein. Transgenic seeds were plated on a solid modified MS medium containing $1 \mu \mathrm{M}$ 17- $\beta$-Estradiol (Sigma-Aldrich E2257) to induce the expression of GFP. Seedlings grew to the 2-4 leaf stage and were then examined with fluorescence microscopy for GFP expression. The presence of the pER8GFP vector in Arabidopsis was also verified by extracting genomic DNA using REDExtract-N-Amp Plant PCR Kit (Sigma-Aldrich) and analyzing on an agarose gel.

\section{Results and Discussion}

\section{(A) Transformation of Agrobacterium strain EHA105 with pER8-GFP}

Verifying the presence of pER8-GFP plasmid in Agrobacterium strain EHA105 was necessary before proceeding with Arabidopsis transformation. Restriction enzyme digest analysis was 
carried out to confirm uptake of pER8-GFP by EHA105. The GFP gene was cloned in between the XhoI and SpeI unique restriction sites of the vector by Zuo et al. In order to confirm uptake of pER8-GFP, the plasmid isolated from EHA105 was cut with XhoI and SpeI restriction enzymes. In addition, the original untransformed pER8-GFP vector and pER8-GFP isolated from EHA105 was cut with $\mathrm{XhoI}$ (single digest) to linearize the vector. Two fragments were expected in the double digest of the vector; a 732 base pair fragment which represents the GFP insert and a second fragment at a higher molecular weight. In the single digest, we expected to see one band at a high molecular weight because the XhoI enzyme is essentially linearizing the vector. Figure 3 shows the linear DNA for both samples running at about $12.2 \mathrm{~Kb}$ which displays the accurate size of the vector (lanes 2 and 3). The double digest of the pER8-GFP DNA extracted from EHA105 verifies that that the GFP is indeed intact in EHA105 as shown by the presence of a band at approximately $700 \mathrm{bp}$ (lanes 4 and 5).

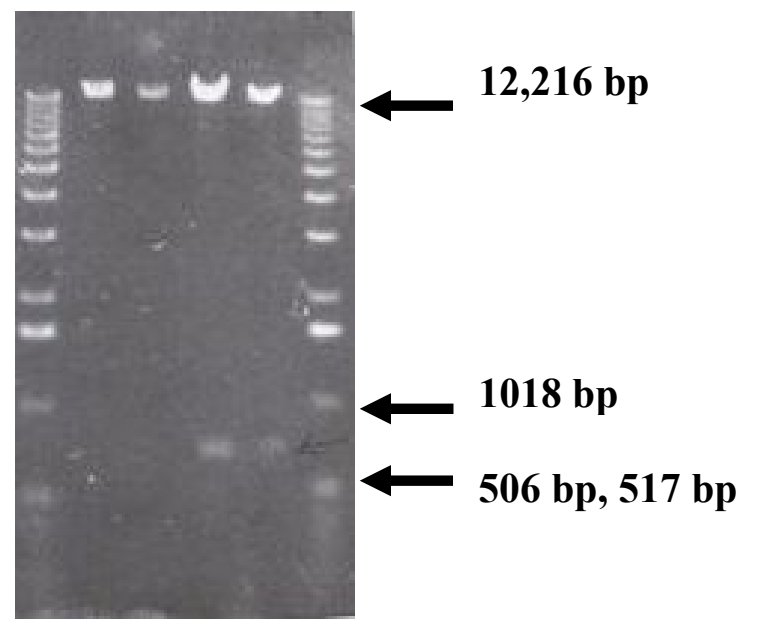

Figure 3. Verification of pER8-GFP plasmid in Agrobacterium strain EHA105.

Lane 1: $1 \mathrm{~Kb}$ Invitrogen DNA ladder

Lane 2: pER8-GFP Transformant (linear DNA)

Lane 3: pER8-GFP Vector Only Untransformed (linear DNA)

Lane 4: pER8-GFP Transformant (GFP excised)

Lane 5: pER8-GFP Vector Only Untransformed (GFP

excised)

Lane 6: $1 \mathrm{~Kb}$ Invitrogen DNA ladder

(B) Selection of hygromycin resistant seedlings. To select transgenic plants, antibiotic resistance is commonly used for selection against sensitive (non-transformed) seedlings. Hygromycin is one of the most popular antibiotics used for selection of transgenic Arabidopsis plants. The problem with hygromycin is that it is quite toxic even to resistant plants causing damaged and deformed seedling growth making it difficult to identify transformants from nontransformants. Initial selection attempts were plagued by this problem while growing on regular MS medium consisting of 0.5 X MS Salts, $2 \%$ Sucrose (w/v), $0.8 \%$ Agar (w/v) and $20 \mu \mathrm{g} / \mathrm{ml}$ hygromycin. Switching to a modified MS medium recipe ${ }^{5}$ allowed clear selection of resistant seedlings (Figure 4). 
(C) GFP reporter assay. Transgenic seeds were plated on modified MS medium plus $1 \mu \mathrm{M}$

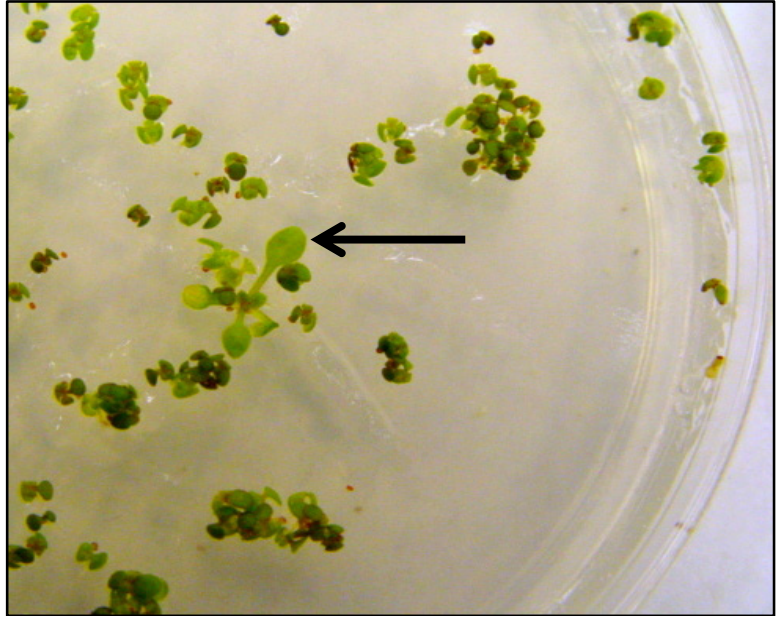

Figure 4. Hygromycin selection of a pER8-GFP transgenic plant. A single transgenic plant carrying hygromycin resistance is indicated by the arrow. It is larger and has a healthy rosette forming. Nontransgenic plants are smaller, deformed and not thriving.
$17-\beta$-Estradiol for induction. They were grown under light exposure $\left(410 \mu \mathrm{moles} / \mathrm{m}^{2} / \mathrm{sec}\right.$ of photons) at $25^{\circ} \mathrm{C}$ during the day and $21^{\circ} \mathrm{C}$ at night for 8 days. Transgenic seeds were also grown in the absence of the estradiol inducer. Transgenic plants were screened by inspection under a conventional fluorescence microscope using Chroma filter set 3200: Ex $425 \mathrm{~nm} \pm 30$ $\mathrm{nm}$, Em $505 \mathrm{~nm} \pm 40 \mathrm{~nm}$. Transgenic seedlings exhibited strong fluorescence in the roots and there was no visible fluorescence from the seedlings grown in the absence of the inducer (Figure 5).
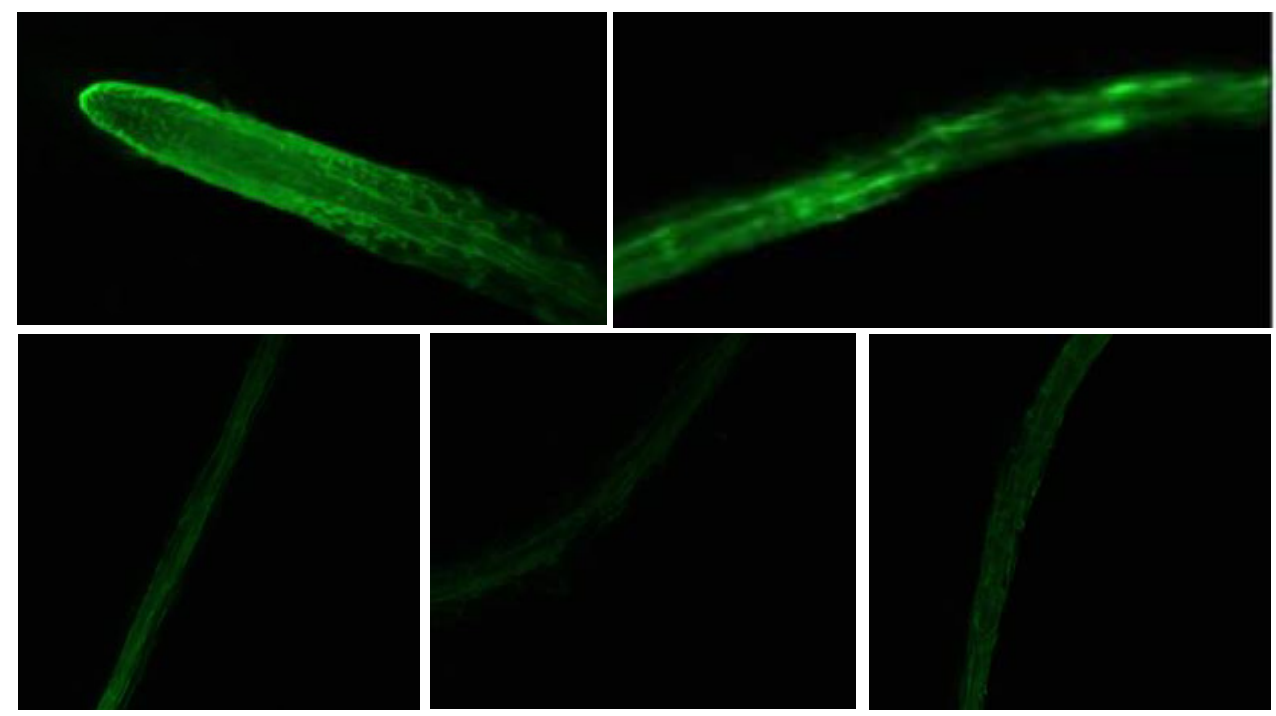

Figure 5. Top: Transformed Arabidopsis induced with estradiol

Bottom: Transformed Arabidopsis-not induced (modified medium + hygromycin), Non-Transformed Arabidopsis (modified medium), Non-Transformed Arabidopsis (modified medium + hygromycin +estradiol)

(D) Arabidopsis genomic DNA analysis. Verification of transformation is commonly achieved by expressing the protein of interest followed by purification, electrophoretic separation, and immunodetection via a Western blot. This is a valid but lengthy process. Here we report on 
transgenic strains by agarose gel analysis of genomic DNA. This is possible because the pER8GFP vector is incorporated into the Agrobacterium genome after transformation.

Transgenic seeds were grown on modified MS medium plus 17- $\beta$-Estradiol for 18 days. Two seedlings were then transferred to PM-15 potting mixture in the ArabiPatch system (LEHLE Seeds) to grow to maturity for seed collection. After six days of growth, a $0.5 \mathrm{~mm}$ diameter leaf sample was removed from the plants for genomic analysis. DNA was extracted using REDExtract-N-Amp Plant PCR Kit (Sigma-Aldrich) instructions.

PCR primers were designed to amplify the GFP portion of the pER8-GFP vector and produce a 323 base product. Three primer sets were ordered (IDT Technologies) and successfully tested on purified plasmid. $1 \mu \mathrm{l}$ and $4 \mu \mathrm{l}$ leaf extracts were tested in PCR using the following primers at a final concentration of $0.5 \mu \mathrm{M}$ :

Forward Primer: 5' - TCAAGgACGACGgGAACTACAAGA - 3'

Reverse Primer: 5' - AgggCAgattgtgtggaCAggtaA - 3'

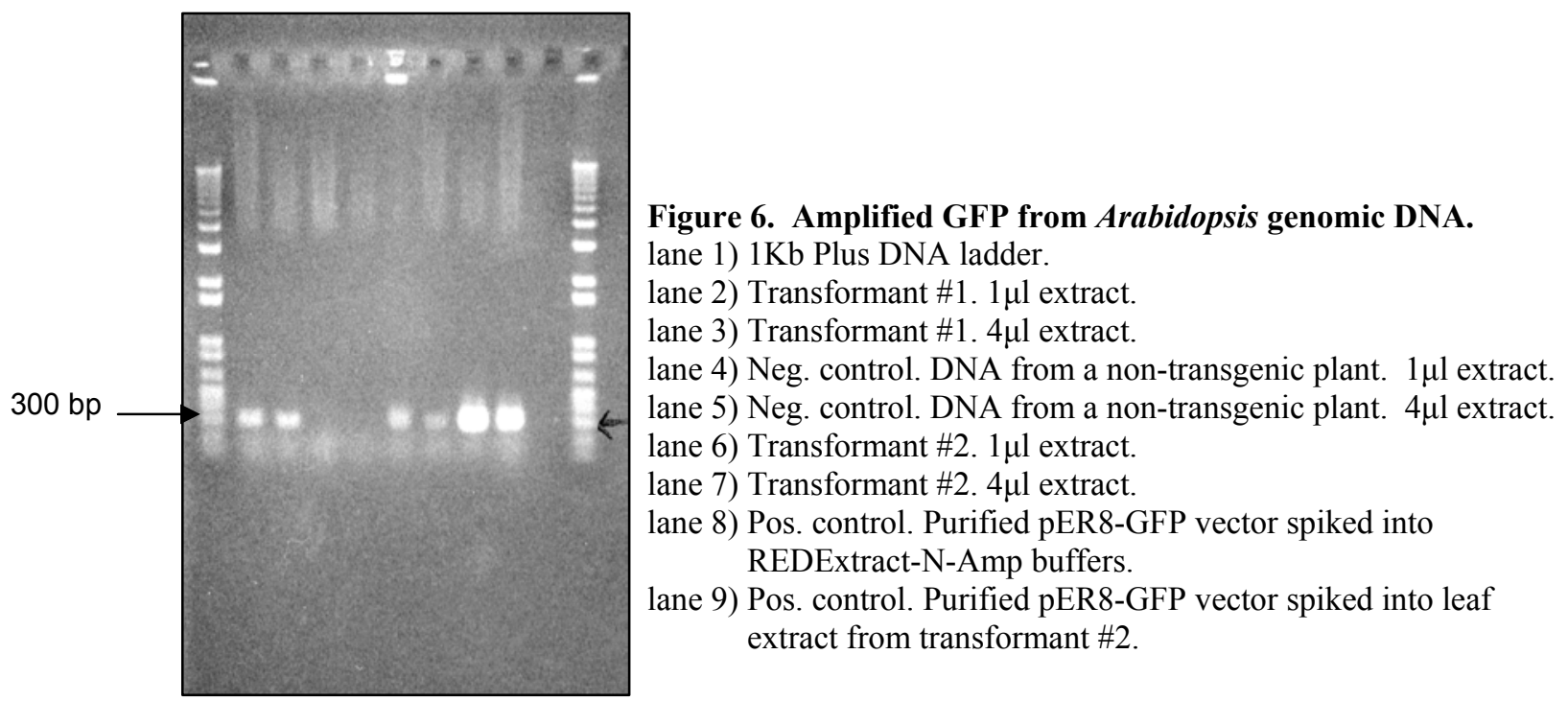

After PCR the amplified DNA was loaded directly onto a 1\% TAE agarose gel and run for 1 hour at $70 \mathrm{mV}$. The DNA was stained with ethidium bromide and visualized under UV light. 
All three plants tested showed strong bands at $\sim 300 \mathrm{bp}$ as measured by a $1 \mathrm{~Kb}$ Plus DNA ladder (Invitrogen) (Figure 6).

\section{Conclusion}

This work demonstrates the feasibility of genetically modified seedling plants as chemical sensors. In a one year time frame we were able to demonstrate the introduction of foreign genes into seedling plants and expression of the reporter gene, GFP, when induced by the presence of estrogen. If follow on funding is obtained, the greatest challenge will be the successful inoculation of mature plants. We used Agrobacterium sp. mediated transformation to introduce the plasmids into the plants. We demonstrated the feasibility of using inoculated plants as chemical sensors by exposing the plants to varying concentrations of estrogen via moisture in the soil. The timed response of the plants to the target analyte was monitored by measuring green

fluorescence of the leaves and roots upon exposure to an ultraviolet (UV) lamp and laser excitation under a light microscope. 


\section{References}

1. Arazi et al. J. Biotech. (2001) 87, 67-82.

2. Tang and Newton, J. Exp. Botany, (2004) 55, 1499-1508.

3. Zuo et al. The Plant Journal (2000) 24(2), 265-273.

4. Plant Molecular Biology Reporter 22: 63-70, March 2004C 2004 International Society for Plant Molecular Biology. Printed in Canada.

5. Biotechniques, (2003) 34, 28-30. 


\begin{tabular}{lll}
\multicolumn{2}{c}{ Distribution } & \\
1 & MS 0123 & Donna Chavez, 1011 \\
1 & MS 1425 & Stephen Casalnuovo, 1714 \\
5 & MS 0892 & Susan Brozik, 1714 \\
1 & MS 0892 & Monica Manginell, 1714 \\
1 & MS 0892 & Dulce Arango, 1714 \\
1 & MS 1425 & Patricia Dolan, 1714 \\
1 & MS 1425 & Jason Harper, 1714 \\
2 & MS 9018 & Central Technical Files, 8944 \\
2 & MS 0899 & Technical Library, 4536
\end{tabular}

